\title{
Characteristics of Static and Dynamic Balance Abilities in Competitive Swimmers
}

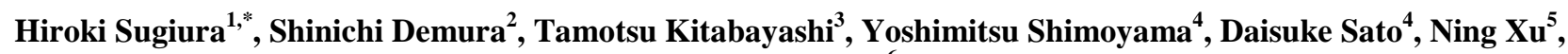 \\ Yuko Asakura ${ }^{6}$ \\ ${ }^{1}$ Department of Industrial Business and Engineering, Fukui University of Technology, Fukui, Japan \\ ${ }^{2}$ Graduate School of Natural Science and Technology, Kanazawa University, Ishikawa, Japan \\ ${ }^{3}$ Faculty of Science Division, Tokyo University of Science, Tokyo, Japan \\ ${ }^{4}$ Department of Health and Sports Sciences, Niigata University of Health and Welfare, Nigata, Japan \\ ${ }^{5}$ Graduate School of Human and Socio-Environmental Studies, Kanazawa University, Ishikawa, Japan \\ ${ }^{6}$ Human and Socio-Environmental Studies, Kanazawa University, Ishikawa, Japan \\ *Corresponding author: sugiura@fukui-ut.ac.jp
}

Received July 21, 2014; Revised November 08, 2014; Accepted November 11, 2014

\begin{abstract}
Competitive swimmers may have inferior balance because antigravity strength exertion, which is used to stand, is not often necessary in the water. This study concerns the ability to stand with the manipulating and supporting legs and their laterality by examining 16 male competitive swimmers (age: $19.4 \pm 1.0$ years, career: $13.7 \pm 2.1$ years) and 16 male general university students (age: $20.6 \pm 1.2$ years). Static balance and dynamic balance were evaluated by the center sway of foot pressure and stability on an unstable stool, respectively. The total path length, mean path length, maximal amplitude rectangle, root mean square area, and outline area for the former and the fluctuation index for the latter were selected as evaluation parameters. The results of a two-way ANOVA (group $\times$ leg) showed no significant difference in both the group and leg factors for static balance parameters. In contrast, the dynamic balance parameter showed a significant difference in both. Stability on an unstable stool was higher in the swimmer group than in the general student group and in the manipulating leg than in the supporting leg in both groups. In conclusion, dynamic balance while standing with the manipulating or supporting leg is superior in competitive swimmers, unlike static balance assessed by the center sway of foot pressure. In addition, dynamic balance in the manipulating leg is superior to that in the supporting leg for both groups.
\end{abstract}

Keywords: static balance, dynamic balance, competitive swimmers

Cite This Article: Hiroki Sugiura, Shinichi Demura, Tamotsu Kitabayashi, Yoshimitsu Shimoyama, Daisuke Sato, Ning Xu, and Yuko Asakura, "Characteristics of Static and Dynamic Balance Abilities in Competitive Swimmers." American Journal of Sports Science and Medicine, vol. 2, no. 6 (2014): 208-211. doi: 10.12691/ajssm-2-6-1.

\section{Introduction}

Balance ability is one of the most important physical fitness factors. It is mainly used while standing and has a close relationship with factors, such as the visual system [1], the vestibular system [2], somatic sensation [3], and leg strength [4]. In addition, balance ability is classified into static and dynamic balance. The former is the ability to stabilize the center of gravity (COP) within a supporting base during static standing, and the latter is one to move it in a new supporting base when being interfered with stability or to maintain stable posture within a supporting base by body movement [5].

It is very important to have superior balance for high performances because many competitive sports are performed in a standing position. Antigravity muscles are involved in maintaining the standing posture [6]. However, the exertion is not always necessary when swimming due to effects of buoyancy [7]. Hence it is hypothesized that competitive swimmers who trained in water for many years have inferior antigravity muscles compared to other competitors [8]. Thus, they are inferior in static and dynamic balance abilities related to antigravity strength.

On the other hand, Noguchi et al. [9] reported that laterality is found in the dynamic balance ability to stand on one leg in general male university students. Laterality means the side of the body people prefer to use in daily activities. Until now, laterality has mostly been studied in upper limbs $[10,11,12]$. However, in the case of lower limbs, Demura et al. [14] reported that people prefer to use a specific leg when hopping on one leg or kicking a ball. Swimming repeats symmetrical movement, and both legs are used equally. Therefore, laterality may not be seen in the balance ability to stand on one leg in competitive swimmers, who have practiced in water for many years.

This study examines the difference in static and dynamic balance abilities and their laterality between competitive swimmers and general university students.

\section{Methods}




\subsection{Participants}

Participants included 16 male competitive swimmers (age: $19.4 \pm 1.0$ years, height: $172.0 \pm 5.7 \mathrm{~cm}$, weight: $65.4 \pm 4.8 \mathrm{~kg}$ ) with swimming careers longer than ten years (career: $13.7 \pm 2.1$ years) and a history of participation at the national level, and 16 healthy male general university students (age: $20.6 \pm 1.2$ years, height: $173.3 \pm 5.5 \mathrm{~cm}$, weight: $68.6 \pm 8.9 \mathrm{~kg}$ ). Nagasawa et al. [13] classified the subjects legs as manipulating leg (used to kick) and supporting leg (used to support the body when kicking a ball) using one item (Which is the leg used to kick a ball?) of the dominant leg survey by Demura et al. [14]. This study employs the same classification. The purpose and procedure of this study were explained to all participants and informed consent was obtained. The present experimental protocol was approved by the Ethics Committee on Human Experimentation of Faculty of Human Science, Kanazawa University (Ref. No. 2012-06).

\subsection{Static Balance}

Static balance has been assessed by measuring the center sway of body gravity while standing [15,16,17]. A Gravicorder G5500 (Anima, Japan) was used to measure foot pressure in this study. This device calculates the center of foot pressure of vertical loads using values of three vertical load sensors put on the peak of an isosceles triangle on a level surface. The center of foot pressure for $30 \mathrm{~s}$ was measured twice with each leg, with a one-minute rest between trials. The representative value was taken from the second trial. Data sampling frequency was recorded at $20 \mathrm{~Hz}[16,17]$. The total path length (length of the center of the foot pressure path), mean path length (mean length of the center of the foot pressure path), maximal amplitude rectangle (area surrounding the maximal amplitude rectangle for each axis), root mean square area (area of the circle creating the actual effective radius value), and outline area (area surrounding the maximal outer bailey for body-sway path) were selected as evaluation parameters for an examination by the equilibrium standardization committee [18]. A larger value in any parameter was judged to be inferior in static balance.

\subsection{Dynamic Balance}

Ogaya et al. [19], Noguchi et al. [9], and Ogaya et al. [20] assessed dynamic balance by testing stability while standing on an unstable stool. The DYJOC Board Plus (SAKAImed, Japan) was used to evaluate stability during a one-leg stand on an unstable stool in this study. This device, in which the bottom of a ship-shaped boss is attached to the central part of the back of a flat board, can slant up to 12 degrees backward and forward and seven degrees to the right and left. The built-in sensor during the one-leg stand on the board perceives gradients of anteroposterior and right-left directions, and measurement data were calculated. The one-leg stand for $30 \mathrm{~s}$ was measured three times with each leg, with a one-minute rest between trials. The value from the third trial was used in the study. Data sampling frequency was recorded at $40 \mathrm{~Hz}$ $[9,19,20]$. The fluctuation index in reference to a report of Ogaya et al. [19] was selected as a parameter. Because this is a mean of absolute values of the inclined angles during measurement, a larger value is judged to be inferior in dynamic balance.

\subsection{Statistical Analysis}

The mean differences of static and dynamic balance parameters were tested by a two-way ANOVA (group $\times$ leg). When significant interactions or mean effect was found, a multiple comparison test was conducted using Tukey's Honestly Significant Difference (HSD) method for multiple comparisons. The significance level in this study was set at $\mathrm{p}<0.05$.

\section{Results}

Table 1 shows the statistics and results of the two-way ANOVA (group $\times$ leg) of static balance parameters. No significant difference was found for the parameters.

Table 2 shows the statistics and results of the two-way ANOVA (group $\times$ leg) of dynamic balance parameters. A significant difference was not found for interaction, but for the main effect of group and leg factors. A multiple comparison test showed that the fluctuation index was lower in the swimmer group than in the general student group for both legs, and for the manipulating leg than for the supporting leg in both groups. In addition, effect sizes for group and leg factors were large $\left(\eta^{2}=0.26,0.15\right)$.

\begin{tabular}{|c|c|c|c|c|c|c|c|c|c|c|c|c|c|}
\hline & & \multicolumn{4}{|c|}{ Manipulating leg (Ma) } & \multicolumn{4}{|c|}{ Supporting leg (Sup) } & & \multirow{2}{*}{$\mathrm{F}$} & \multirow{2}{*}{$\eta^{2}$} & \multirow{2}{*}{$\mathrm{p}$} \\
\hline & & $\mathrm{M}$ & SD & MAX & MIN & $\mathrm{M}$ & SD & MAX & MIN & & & & \\
\hline \multirow{3}{*}{ Total path length (cm) } & G1 & 105.3 & 21.6 & 142.5 & 59.4 & 106.0 & 19.9 & 135.0 & 70.4 & F1 & 0.26 & 0.01 & 0.62 \\
\hline & G2 & 108.0 & 25.5 & 174.4 & 72.3 & 110.9 & 21.3 & 143.9 & 74.9 & F2 & 0.38 & 0.01 & 0.54 \\
\hline & & & & & & & & & & IN & 0.13 & 0.00 & 0.72 \\
\hline \multirow{3}{*}{ Mean path length (cm/s) } & G1 & 3.8 & 1.0 & 5.6 & 2.0 & 3.5 & 0.7 & 4.5 & 2.4 & F1 & 0.05 & 0.00 & 0.82 \\
\hline & G2 & 3.7 & 1.1 & 7.5 & 2.4 & 3.8 & 0.8 & 5.4 & 2.5 & F2 & 0.89 & 0.03 & 0.35 \\
\hline & & & & & & & & & & IN & 1.21 & 0.04 & 0.28 \\
\hline \multirow{3}{*}{ Maximal amplitude rectangle $\left(\mathrm{cm}^{2}\right)$} & G1 & 11.4 & 4.1 & 23.3 & 6.2 & 10.9 & 3.3 & 17.5 & 4.8 & F1 & 3.14 & 0.09 & 0.09 \\
\hline & G2 & 14.0 & 3.6 & 21.7 & 9.1 & 18.1 & 19.8 & 94.2 & 9.5 & F2 & 0.50 & 0.02 & 0.49 \\
\hline & & & & & & & & & & IN & 0.78 & 0.03 & 0.38 \\
\hline \multirow{3}{*}{ Root mean square area $\left(\mathrm{cm}^{2}\right)$} & G1 & 2.6 & 1.0 & 5.6 & 1.3 & 2.8 & 1.0 & 5.3 & 1.3 & F1 & 1.03 & 0.03 & 0.32 \\
\hline & G2 & 3.8 & 3.5 & 16.7 & 0.9 & 3.1 & 2.0 & 10.1 & 1.0 & F2 & 0.44 & 0.01 & 0.51 \\
\hline & & & & & & & & & & IN & 2.49 & 0.08 & 0.13 \\
\hline \multirow{3}{*}{ Outline area $\left(\mathrm{cm}^{2}\right)$} & G1 & 11.3 & 12.6 & 57.5 & 2.9 & 10.3 & 9.5 & 40.4 & 2.5 & F1 & 0.02 & 0.00 & 0.89 \\
\hline & G2 & 9.4 & 4.0 & 18.9 & 4.4 & 13.2 & 15.8 & 73.1 & 5.4 & F2 & 0.53 & 0.02 & 0.47 \\
\hline & & & & & & & & & & IN & 1.55 & 0.05 & 0.22 \\
\hline
\end{tabular}

G1: Swimmers ( $=16)$, G2: General students $(n=16)$, Ma: Manipulating leg, Sup: Supporting leg

F1: Group (G1, G2) , F2: Leg (Ma, Sup) , IN: Interaction 
Table 2. Differences in dynamic balance parameter among groups

\begin{tabular}{|c|c|c|c|c|c|c|c|c|c|c|c|c|c|c|}
\hline & & \multicolumn{4}{|c|}{ Manipulating leg (Ma) } & \multicolumn{4}{|c|}{ Supporting leg (Sup) } & & \multirow{2}{*}{$\mathrm{F}$} & \multirow{2}{*}{$\eta^{2}$} & \multirow{2}{*}{$\mathrm{p}$} & \multirow{2}{*}{ Tukey's HSD } \\
\hline & & M & SD & MAX & MIN & M & SD & MAX & MIN & & & & & \\
\hline \multirow{3}{*}{$\begin{array}{l}\text { Total angle } \\
\text { fluctuation level }\end{array}$} & G1 & 277.2 & 63.9 & 388.4 & 155.2 & 302.4 & 81.5 & 450.1 & 191.8 & $\mathrm{~F} 1$ & $10.64^{*}$ & 0.26 & 0.00 & [Both legs] \\
\hline & \multirow[t]{2}{*}{ G2 } & \multirow[t]{2}{*}{359.6} & \multirow[t]{2}{*}{76.7} & \multirow[t]{2}{*}{478.6} & \multirow[t]{2}{*}{206.8} & \multirow[t]{2}{*}{394.6} & \multirow[t]{2}{*}{100.5} & \multirow[t]{2}{*}{634.8} & \multirow[t]{2}{*}{221.8} & F2 & $5.21^{*}$ & 0.15 & 0.03 & $\begin{array}{c}\mathrm{G} 1<\mathrm{G} 2 \\
\text { [Both groups] }\end{array}$ \\
\hline & & & & & & & & & & IN & 0.14 & 0.00 & 0.71 & Ma < Sup \\
\hline
\end{tabular}

G1: Swimmers ( $=16)$, G2: General students $(n=16)$, Ma: Manipulating leg, Sup: Supporting leg

F1: Group (G1, G2), F2: Leg (Ma, Sup), IN: Interaction

$*: \mathrm{p}<0.05$.

\section{Discussion}

Static balance has been assessed by the center sway of body gravity in a standing posture [15]. Tanaka et al. [15], Kitabayashi et al. [16], and Matsuda et al. [17] assessed static balance by the center of foot pressure when participants stood on a force plate for a specific amount of time. In this study, a similar method was used to evaluate the participants' static balance. As a result, no significant difference was found in all static balance parameters between the swimmers and general student groups and between the manipulating and supporting legs. Competitive swimmers train in the water with buoyancy for a long time. Hence, it is hypothesized that they have inferior static balance, important for the standing posture, due to the little use of antigravity muscles as compared to the general students. However, this hypothesis was rejected and no significant difference was found between both groups.

Hahn et al. [21] reported that the one-leg stand time with closed eyes showed no significant difference among soccer players, handball players, basketball players, badminton players, tennis players, gymnasts, and swimmers. In addition, Matsuda et al. [17] examined static balance among soccer players, basketball players, swimmers, and non-athletes, and reported no significant difference between swimmers and non-athletes. University competitive swimmers in this study had swimming careers longer than ten years and competition history at the national level, and the non-athletes were general university students of similar ages. It is considered that competitive swimmers could perform the one-leg stand easily for $30 \mathrm{~s}$, similar to the general students, if the standing posture was not disturbed during the measurement.

Matsuda et al. [17] reported that laterality was not found in static balance for the one-leg stand in soccer players, basketball players, swimmers, and non-athletes. No significant difference was found in each static balance parameter between the swimmers and general students in this study. Many activities of daily life, such as walking, ascending, and descending stairs, and standing up, necessitate the use of both legs. Swimming is also an exercise that repeats symmetrical movement, and both legs are used equally; therefore, laterality was not found.

The one-leg stand on an unstable moving stool such as the DYJOC board forced subjects to maintain a stable posture under a peculiar condition and demanded the ability to retain the stable posture by using the body's core and legs. The present results show that the swimmer group is superior in the dynamic balance of each leg than the general student group. Davlin [22] also reported that swimmers are superior to general students in dynamic balance. Seifert et al. [23] reported that expert swimmers are superior in their limb coordination. According to Shimojyo et al. [24], swimmers should attach great importance to the following somatosensory factors: resistance of water, joint angle, physical position, and exercise efficiency. To reduce swimming times, it is important to reduce water resistance, and somatosensory function is demanded in the water. Because the present competitive swimmers have experience at the national level, they are considered to have superior somatosensory function.

In addition, Liao and Lin [25] reported that a strong relationship was found between the center of mass displacement and the angular displacement of the ankles. Demura and Matsuura [26] and Demura et al. [27] reported that ankle flexibility is important for kicking in swimming. In short, it is inferred that the present competitive swimmers have greater ankle flexibility than the general students. Also, even in the case of a largely inclined wobble board, they could easily maintain a stable posture by coordinating their ankle joints.

Noguchi et al. [9] examined the laterality of dynamic balance in general university male students and reported that the manipulating leg was superior to the supporting leg. The result for the general students in this study is similar to that in Noguchi et al. [9]. In addition, it was found that the dynamic balance of the manipulating leg is superior in competitive swimmers to that of the supporting leg. Both legs are used equally in daily life. However, in the case of special movement, such as kicking a ball, one leg is preferably used and the other leg contributes to maintaining a stable posture and allowing for easier control of a ball. In short, the role of each leg is different. When kicking a ball, it was shown that the manipulating and supporting legs present similar results for general students and competitive swimmers who repeatedly practice symmetric movements. From these results, the manipulating leg with high operability may have superior ability to maintain a stable posture on the continuously changing stool stability to the supporting leg.

\section{Conclusion}

No significant difference was found in the static balance assessed by the center of foot pressure between competitive swimmers and general students and between manipulating and supporting legs in both groups. However, dynamic balance is superior in competitive swimmers than in general students and in the manipulating leg to the supporting leg.

\section{Acknowledgement}

Research funds were not provided by any institution. 


\section{Conflict of Interest Statement}

None.

\section{References}

[1] Lord, S. R., "Visual risk factors for falls in older people," Age and Ageing; 35 (2), ii42-ii45. 2006.

[2] Choy, N.L., Johnson, N., Treleaven, J., Jull, G., Panizza, B. and Brown-Rothwell, D., "Balance, mobility and gaze stability deficits remain following surgical removal of vestibular schwannoma (acoustic neuroma): an observational study,” Australian Journal of Physiotherapy, 52 (3). 211-216. 2006.

[3] Lord, S.R., Clark, R.D., and Webster, I.W., "Postural stability and associated physiological factors in a population of aged persons," The Journal of Gerontology, 46 (3). 69-76. 1991.

[4] Aniansson, A., Rundgren, A., and Sperling, L., "Evaluation of functional capacity in activities of daily living in 70-year-old men and women," Scandinavian Journal of Rehabilitation Medicine, 12 (4). 145-154. 1980.

[5] Takeshima, N., and Rogers, M.E., Theory and practice of the balance exercise for the fall prevention, Nap, Tokyo, 2010, 11-18.

[6] Fitzpatrick, R., Rogers, D.K., and McCloskey, D.I., "Stable human standing with lower-limb muscle afferents providing the only sensory input,” The Journal of Physiology, 480. 395-403. 1994.

[7] Taguchi, M., Takesita, K., Takagi, H., and Morihata, M., "Muscle strength exhibition characteristics by the sporting events," Training Science, 4 (1). 84-91. 1992.

[8] Yamaji, S., and Demura, S., "Differences among competitive sports in force output of various leg muscle contractions," Gazzetta Medica Italiana, 171 (6). 713-719. 2012.

[9] Noguchi, T., Demura, S., and Nakagawa, T., "Posture stability during a one-leg stance on an unstable moving platform and its relationship with each leg,” Perceptual and Motor Skills, 116 (2) 555-563. 2013.

[10] Nagasawa, Y., Demura, S., Yamaji, S., Kobayashi, H., and Matsuzawa, J., "Ability to coordinate exertion of force by the dominant hand: comparisons among university students and 65- to 78-year-old men and women," Perceptual and Motor Skills, 90. 995-1007. 2000

[11] Noguchi, T., Demura, S., and Aoki, H., "Superiority of the dominant and nondominant hands in static strength and controlled force exertion," Perceptual and Motor Skills, 109 (2). 339-346. 2009.

[12] Kubota, H., Demura, S., and Kawabata, H., "Laterality and agelevel differences between young women and elderly women in controlled force exertion (CFE)," Archives of Gerontology and Geriatrics, 54 (2). e68-e72. 2012.
[13] Nagasawa, Y., Demura, S., Matsuda, S., Uchida, Y., and Demura, T., "Effect of differences in kicking legs, kick directions, and kick skill on kicking accuracy in soccer players," Journal of Quantitative Analysis in Sports, 7 (4). Article 9. 2011.

[14] Demura, S., Sato, S., and Sugiura, H., "Lower limb laterality characteristics based on the relationship between activities and individual laterality,” Gazzetta Medica Italiana, 169 (5). 181-191. 2010.

[15] Tanaka, T., Noriyasu, S., Ino, S., Ifukube, T., and Nakata, M., "Objective method to determine the contribution of the great toe to standing balance and preliminary observations of age-related effects," IEEE Transactions on Neural Systems and Rehabilitation Engineering, 4 (2). 84-90. 1996.

[16] Kitabayashi, T., Demura, S., and Noda, M., "Examination of the factor structure of center of foot pressure movement and crossvalidity,” Journal of Physiological Anthropology and Applied Human Science, 22 (6). 265-272. 2003.

[17] Matsuda, S., Demura, S., and Uchiyama, M., "Centre of pressure sway characteristics during static one-legged stance of athletes from different sports,” Journal of Sports Sciences, 26 (7). 775-779. 2008.

[18] Examination of equilibrium standardization committee, "The standardization of the equilibrium function test," Equilibrium Research, 65 (6). 468-503. 2006

[19] Ogaya, S., Ikezoe, T., Tsuboyama, T., and Ichihashi, N., "Postural control on a wobble board and stable surface of young and elderly people," Physical Therapy science, 24 (1). 81-85. 2010.

[20] Ogaya, S., Ikezoe, T., Soda, N., and Ichihashi, N., "Effects of balance training using wobble boards in the elderly," The Journal of Strength and Conditioning Research, 25 (9). 2616-2622. 2011.

[21] Hahn, T., Foldspang, A., Vestergaard, E., and Ingemann-Hansen, T., "One-leg standing balance and sports activity," Scandinavian Journal of Medicine \& Science in Sports, 9 (1). 15-18. 1999.

[22] Davlin, C.D., "Dynamic balance in high level athletes," Perceptual and Motor Skills, 98 (3). 1171-1176. 2004.

[23] Seifert, L., Leblanc, H., Chollet, D., and Delignières, D., "Interlimb coordination in swimming: effect of speed and skill level," Human Movement Science, 29 (1). 103-113. 2010.

[24] Shimojo, H., Sengoku, Y., Tsubakimoto, S., and Takagi, H., "The important kinesthesia for enhancement of swimming skill in college swimmers," Journal of Physical Education Health and Sport Sciences, 57. 201-213. 2012

[25] Liao, C.F., and Lin, S.I., "Effects of different movement strategies on forward reach distance," Gait and Posture, 28 (1). 16-23. 2008.

[26] Demura, S., and Matsuura, Y., "A flexibility test battery for college male swimmers," The Japanese Journal of Physical Fitness and Sports Medicine, 32 (2). 94-102. 1982.

[27] Demura, S., Matsuzawa, J., Naka, H., and Kita, I., "Physica chracteristics in well-trained young swimmers," The Japanese Journal of Physical Fitness and Sports Medicine, 40 (3). 278-287. 1991. 